\title{
Adenocarcinoma de vulva: un reporte de caso tipo glándula mamaria
}

\begin{abstract}
*Correspondencia:
hector_montes93@hotmail.com
\end{abstract}

Dirección: Av. Samborondón 5, Samborondón, Guayaquil-Ecuador. CP092301. Teléfono [593] (04) 500-0950

\section{Conflicto de intereses: LoS autores declaran no tener conflictos de intereses.}

Recibido: 16 de Septiembre 2021 Aceptado: 27 de Noviembre, 2021 Publicado: 20 de Diciembre, 2021 Editor: Dr. Felipe Campoverde

\section{Membrete bibliográfico:}

Alarcón C, Rivadeneira N, Montes $\mathrm{H}$. Adenocarcinoma de vulva: un re porte de caso tipo glándula mama ria. Rev. Oncol. Ecu 2021;31(3):243-250.

DOI: https://doi.org/10.33821/567

Copyright Alarcón C, et al. Este artículo es distribuido bajo los términos de Creative Commons Attribution License BY-NCSA 4.0, el cual permite el uso y re distribución citando la fuente y al autor original.

\section{Adenocarcinoma of the vulva: a case study type} mammary gland

\section{Carlos Alarcon Andrade1, Nery Rivadeneira Santana1, Héctor Montes Lainez*2,3 (DD}

1. Servicio de Ginecología Oncológica, Instituto Oncológico Nacional "Dr- Juan Tanca Marengo" SOLCA-Guayaquil, Ecuador.

2. Departamento de postgado de Cirugía, Universidad de Especialidades Espíritu Santo, Samborondón, Guayaquil, Ecuador

3. Servicio de Cirugía Oncológica, Instituto Oncológico Nacional "Dr- Juan Tanca Marengo" SOLCA-Guayaquil, Ecuador

\section{Resumen}

Introducción: Los tumores malignos de vulva son tumores raros del tracto genital femenino, que representan menos del $1 \%$ de los tumores malignos del sexo femenino, cerca del 95\% de estos son carcinoma escamo celulares, los adenocarcinomas son el tipo histológico menos frecuente, con 36 casos reportados hasta el año 2020.

Caso Clínico: Presentamos el caso de una mujer de 36 años con antecedente de histerectomía total por miomatosis, con cuadro clínico de 1 año de evolución caracterizado por una masa indolora en labio mayor izquierdo la cual se somete a biopsia que reporta adenocarcinoma motivo por el cual se somete a vulvectomia radical, en resultado patológico definitivo se confirma el diagnostico de adenocarcinoma de vulva tipo glándula mamaria.

Evolución: Se realiza vulvectomía radical + vaciamiento ganglionar inguino-iliaco bilateral. El estudio histo-patológico definitivo reveló adenocarcinoma de vulva tipo glándula mamaria pobremente diferenciado G3, Unifocal, con metástasis en 22/25 ganglios derechos y 30/30 izquierdos. Un estudio reportó recidiva local extensa, metástasis ganglionares pélvicas, retroperitoneales, mediastinales, axilares, cervicales y pulmonar. Se inició Paclitaxel $137 \mathrm{mg}$ semanal. Se completa quimioterapia hasta 11 ra semana posterior a lo cual presentó infección de lecho tumoral y fallece.

Conclusión: En el presente caso el curso clínico de adenocarcinoma de vulva tipo glándula mamaria fue agresivo con una supervivencia inferior a 5 meses.

Palabras claves:

DeCS: Neoplasias de la Vulva, Vulva, Vulvectomía, Informes de Casos, Oncología Médica.

DOI: $10.33821 / 567$ 


\section{Abstract}

Introduction: Malignant tumors of the vulva are rare tumors of the female genital tract, which represent less than $1 \%$ of malignant tumors of the female sex, about $95 \%$ of these are squamous cell carcinoma, adenocarcinomas are the least frequent histological type, with 36 cases reported until 2020.

Clinical Case: We present the case of a 36-year-old woman with a history of total hysterectomy due to myomatosis, with a clinical picture of 1 year of evolution characterized by a painless mass in the left labia majora which undergoes a biopsy that reports adenocarcinoma, which is why undergoes radical vulvectomy, the definitive pathological result confirms the diagnosis of adenocarcinoma of the vulva type mammary gland.

Evolution: Radical vulvectomy + bilateral inguino-iliac lymph node dissection was performed. The definitive histopathological study revealed poorly differentiated G3, Unifocal mammary gland adenocarcinoma of the vulva, with metastases in 22/25 right and 30/30 left lymph nodes. One study reported extensive local recurrence, pelvic, retroperitoneal, mediastinal, axillary, cervical and pulmonary lymph node metastases. Paclitaxel $137 \mathrm{mg}$ weekly was started. Chemotherapy was completed up to the 11 th week after which he presented a tumor bed infection and died.

Conclusion: In the present case, the clinical course of adenocarcinoma of the vulva type of the mammary gland was aggressive with a survival of less than 5 months.

\section{Keywords:}

MESH: Vulvar Neoplasms, Vulva, Vulvectomy, Case Reports, Medical Oncology.

DOI: $10.33821 / 567$

\section{Introducción}

Los tumores malignos de vulva son los menos frecuentes del tracto genital femenino (3 a 4 \%) [1]. Siendo el carcinoma escamocelular el tipo histológico más frecuente (95\%) y asociado en la mayoría de los casos al virus del papiloma humano (VPH) [2].

El adenocarcinoma de vulva representa menos del 1\% del total de cáncer de vulva y de estos, la mitad son secundarios, los adenocarcinomas primarios toman el nombre en función del presunto tejido de origen pudiéndose denominar adenocarcinoma de glándula de Bartholine, de uretra terminal, de glándula sudorípara, tipo glándula mamaria, etc [3], siendo de estos el adenocarcinoma de vulva tipo glándula mamaria el más infrecuente, hasta el 2020 se encontraban descritos en la literatura mundial únicamente 36 casos [4]. Debido a lo raro de esta patología presentamos el siguiente caso clínico.

\section{Caso clínico}

Paciente de sexo femenino de 36 años de edad, quien tenía antecedentes gineco-obstétricos de 2 cesáreas, e histerectomía total por miomatosis uterina 4 años previos a su consulta. El cuadro clínico fue la presencia de masa en labio mayor izquierdo que ha ido en crecimiento 
paulatinamente durante el último año. Se realizó en una institución externa un estudio citológico del tumor, cuyo diagnóstico era compatible con carcinoma escamoso de vulva, motivo por el cual fue derivada y valorada por el servicio de colposcopía en donde se realiza una biopsia de la masa cuyo resultado reporta adenocarcinoma pobremente diferenciado de vulva, y es derivada al servicio de ginecología.

Al examen físico se evidenció una masa de $3 \mathrm{~cm}$ en labio mayor izquierdo en tercio medio, ulcerada y friable, con adenopatías inguinales bilaterales móviles (figura 1). Se realiza RMN donde se evidencia la lesión ya conocida, además de adenopatías inguinales bilaterales (figura 2). Se programa para cirugía radical ya que adenopatías inguinales podrían ser inflamatorias por el antecedente de múltiples biopsias, siendo necesaria linfadenectomía para estadificación.

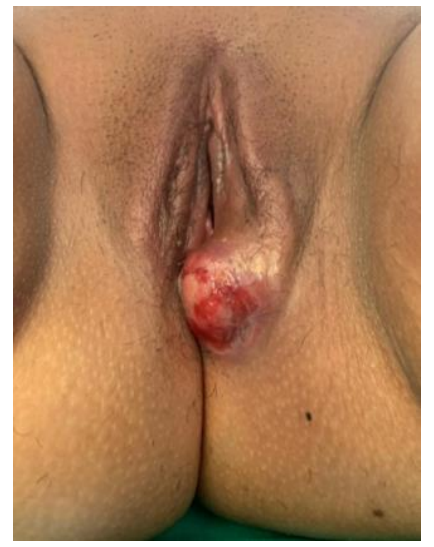

Fiqura 1 Lesión Ulcerada friable dura de tercio inferior de labio mavor

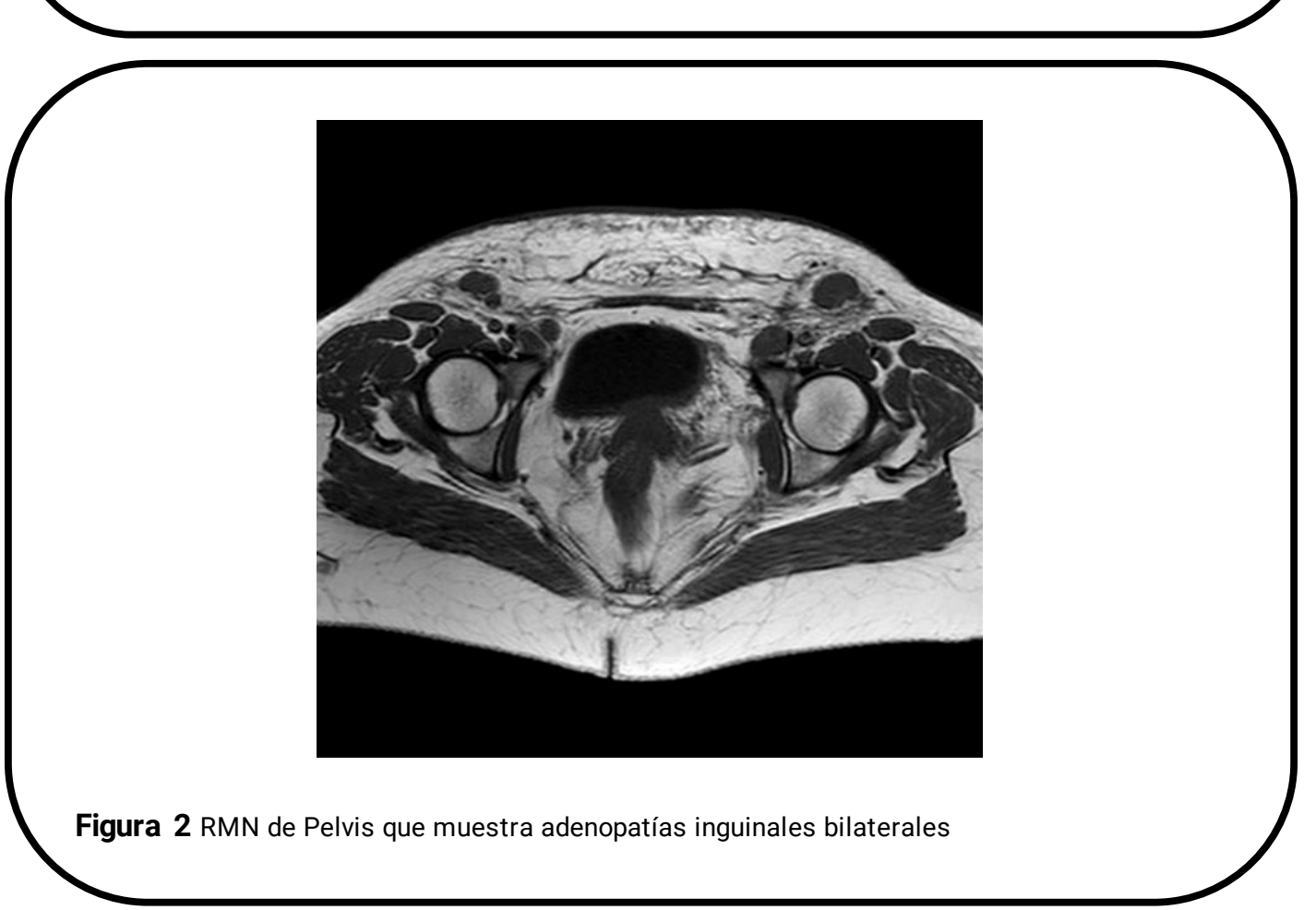




\section{Evolución}

Se realiza vulvectomía radical + vaciamiento ganglionar inguino-iliaco bilateral. El estudio histopatológico definitivo reveló: Adenocarcinoma de vulva tipo glándula mamaria pobremente diferenciado G3, Unifocal, con metástasis en 22/25 ganglios derechos y 30/30 izquierdos. La paciente fue transferida al servicio de oncología clínica en donde se indica la realización de un PetScan que reporta: Recidiva local extensa, metástasis ganglionares pélvicas, retroperitoneales, mediastinales, axilares, cervicales y pulmonar (Figura 3). Se decide el inicio de esquema Quimioterápico, con Paclitaxel 137 mg semanal. Radioterapia no se da por lo avanzado de la enfermedad. Se completa quimioterapia hasta 11ra semana por aparente buena tolerancia, posterior a lo cual presenta complicaciones clínicas (infección de lecho tumoral), es ingresada en la unidad paliativa de la institución y fallece 3 semanas después.

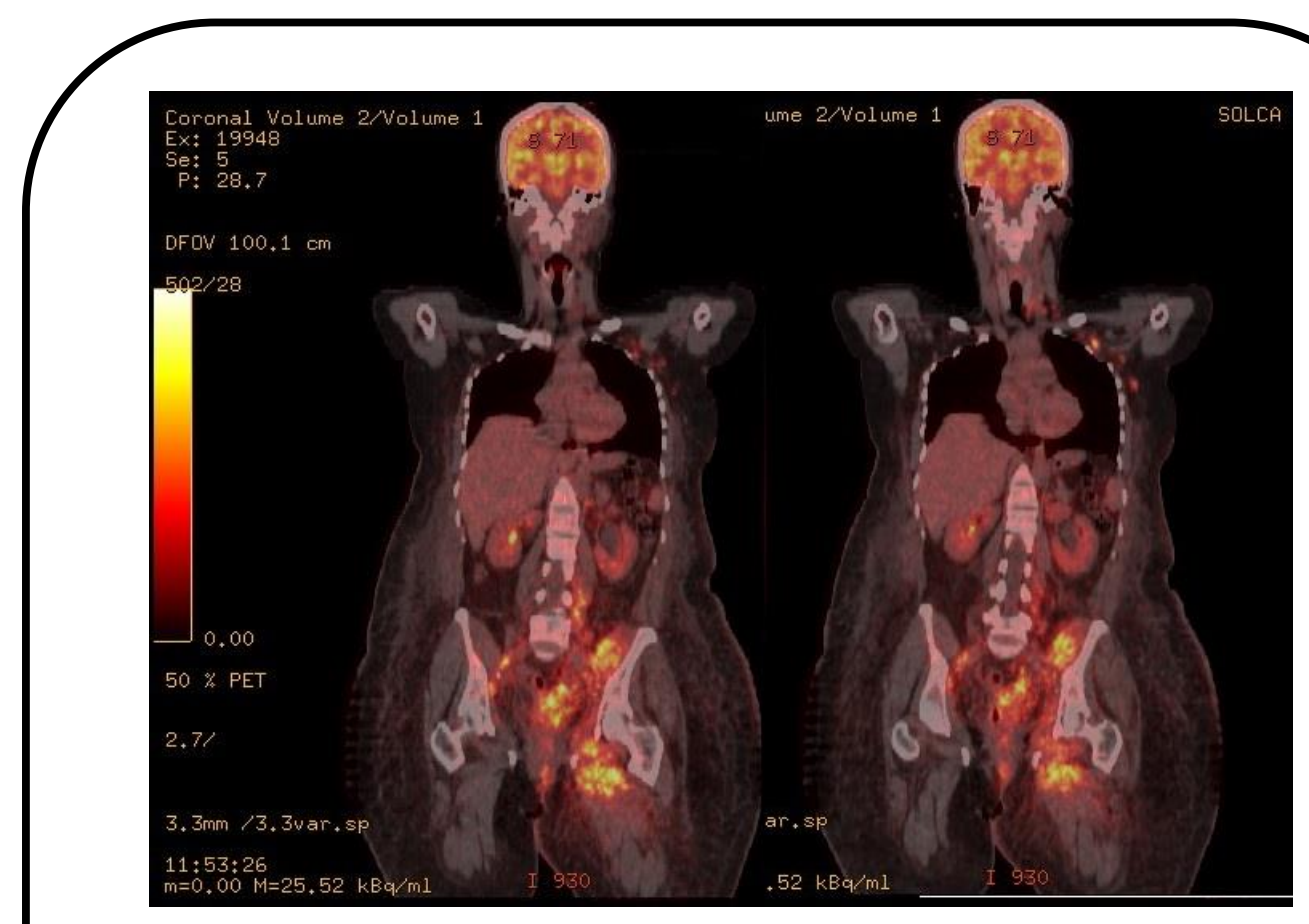

Figura 3 Pet Scan corporal total. Corte Coronal: metástasis ganglionares pélvicas, retroperitoneales, mediastinales, axilares, cervicales y pulmonar

\section{Discusión}

El cáncer de vulva es una patología rara que representa menos del $1 \%$ de todos los tumores malignos en el sexo femenino [1]. Y representan alrededor del 3 al $4 \%$ de todas las neoplasias malignas del tracto genital femenino [2]. Se da generalmente en mujeres postmenopáusicas [3].

En el 2019 se reportaron 6070 casos nuevos en Estados Unidos, lo cual representa el 0.3\% de todos los canceres nuevos en este país [4]. Del 100\% de este tipo infrecuente de cáncer, el 
carcinoma de células Escamosas representa alrededor del $95 \%$, y se encuentra asociado al VPH [드, 6]. El Adenocarcinoma de Vulva representa el 1\% de cáncer de este sitio anatómico, siendo el tipo histológico más raro e infrecuente. En Ecuador hay escasos reportes de esta neoplasia vulvar, todos los descritos siendo carcinoma de células escamosas y ningún reporte de adenocarcinoma [7].

Aproximadamente la mitad de los adenocarcinomas de vulva son secundarios. Los primarios son extremadamente raros y se clasifican nominalmente en función del presunto tejido de origen, fenotipo tumoral o ambos. De esta forma encontramos: Adenocarcinomas de glándula de Bartholine, de uretra terminal, de tipo intestinal (tipo cloacogénico), tipo glándula mamaria, Carcinoma de células claras [].

Las glándulas que se encuentran en la región vulvar se las puede dividir en glándulas sudoríparas y anogenitales tipo glándula mamaria, siendo esta ultima la que se maligniza, e incluso expresan receptores de Estrógeno y Progesterona [8].

Los adenocarcinomas de glándulas sudoríparas, de tipo mamario y enfermedad de paget extramamario comparten una histopatología similar, por lo cual Van der Putter y Van Gorp propusieron un concepto unificador e introdujeron el termino Adenocarcinoma de Vulva tipo Glándula Mamaria para este tipo de tumores en 1994 [8].

La sintomatología más frecuente es el prurito vaginal generalmente de larga data acompañado de tumefacción en el área genital externa asociada a ulceraciones, localizándose más frecuentemente en los labios mayores (40\%) [1].

El diagnóstico se basa en la clínica que tiene que ir acompañada de un diagnóstico por histopatología mediante biopsia, se remienda en toda paciente con lesiones vulvares realizar vulvoscopía con apoyo de ácido acético para identificar lesiones potencialmente malignas, pero en este tipo de adenocarcinoma no es efectivo por lo que se recomienda biopsia con muestra de tejido que incluya tejido subcutáneo. Se recomienda realizar examen de pesquizaje mamario en todo paciente con adenocarcinoma tipo glándula mamaria [3].

Debido a lo rara de esta enfermedad no existe un consenso claro de tratamiento [10]. Hasta el año 2020 solo existían reportados 36 casos en la literatura mundial sobre esta patología [11].

Debido a la histopatología similar a la glándula mamaria se extrapola guías de cáncer de mama a este tipo de cáncer, siendo de principal importancia determinar la inmunohistoquímica del tumor para definir el tratamiento, en los 36 casos reportados en la literatura mundial, la incidencia de Her 2 neu es similar al cáncer de mama (15 al 19\%) [11].

Al igual que es cáncer de mama la piedra angular del tratamiento en los casos reportados constituye en la cirugía [12]. Esta va desde escisión local amplia hasta vulvectomia radical con disección ganglionar inguinofemoral bilateral, pudiéndose acompañar de terapia neoadyuvante o adyuvante [11]. En el caso presentado se realizó cirugía radical, ya que no existía evidencia de metástasis hasta antes de la cirugía. El tratamiento adyuvante se debe guiar con los mismos lineamientos que el cáncer de mama que incluyen radioterapia, quimioterapia basada en antraciclinas y terapia hormonal. La Neoplasia intraepitelial premaligna se ha tratado con éxito con imiquimod local [11].

No existen ensayos clínicos para evaluar la eficacia de la quimioterapia o radioterapia debido al pequeño número de pacientes, por lo que se recomienda el procedimiento de escisión con o sin disección de los ganglios linfáticos, seguida de otras terapias adyuvantes según sea necesario. El uso de Ganglio centinela está indicado [13]. El cáncer de vulva es ideal para el 
Nota del Editor

La Revista Oncología Ecu permanece neutral con respecto a los reclamos jurisdiccionales en mapas publicados y afiliaciones institucionales. mapeo de ganglio centinela debido al drenaje linfático predecible de la vulva, la baja tasa de falsos negativos y la morbilidad reducida [16].

La decisión de realizar neoadyuvancia o cirugía primaria podría extrapolarse a las mismas normas de cáncer de mama (evidencia de invasión locoregional) aunque solo existe un caso de este tipo de cáncer tratado con neadyuvancia con trastazumab [11] con resultados aceptables. Solo existe un trabajo reportado donde no se realizó ningún tipo de resección y se inició radioterapia y quimioterapia con Paclitaxel debido a enfermedad avanzada, pero con pobres resultados [11]. En casi todos los trabajos reportados se ha realizado algún tipo de resección quirúrgica con linfadenectomía, siendo la vulvectomía radical la más común y la realizada en este caso, la evidencia reporta que la adyuvancia mejora la sobrevida de estos pacientes, aquellos que no reciben adyuvancia tienen una sobrevida de 4 meses frente a la mediana de supervivencia de 22 meses que reporta para aquellos que si la recibieron [13].

\section{Conclusiones}

El carcinoma de vulva en general representa una entidad maligna sumamente rara que muchas veces es confundida en la atención inicial, lo cual dificulta el diagnóstico precoz e impide el tratamiento. La cirugía, al igual que en el cáncer de mama, continúa siendo la piedra angular del tratamiento que en combinación con quimioterapia, radioterapia y terapia hormonal postoperatoria han demostrado mejorar la expectativa de supervivencia de este tipo de pacientes.

\section{Abreviaturas}

VEB: virus de Epstein-Barr, VPH: virus del papiloma humano.

\section{Información administrativa}

Archivos Adicionales

Ninguno declarado por los autores.

Agradecimientos

No aplica.

Contribuciones de los autores

Carlos Alarcon Andrade: conceptualización, validación, visualización, metodología, administración de proyecto, escritura: revisión y edición, curación de datos, análisis formal, adquisición de fondos, investigación, recursos, software, redacción -borrador original.

Nery Rivadeneira Santana: conceptualización, validación, visualización, metodología, admin istración de proyecto, escritura: revisión y edición, curación de datos, análisis formal.

Héctor Montes Lainez: conceptualización, análisis formal, adquisición de fondos, investigación, recursos, software, redacción -borrador original.

Todos los autores leyeron y aprobaron la versión final del manuscrito. 
Financiamiento

Los autores realizaron el financiamiento de los gastos incurridos en la presente investigación.

Disponibilidad de datos y materiales

Existe la disponibilidad de datos bajo solicitud a la autora de correspondencia. No se reportan otros materiales.

\title{
Declaraciones
}

Aprobación del comité de ética

No aplica.

Consentimiento para publicación

Se cuenta con el consentimiento de publicación por parte de la paciente.

\section{Conflictos de interés}

Los autores declaran que no tener ningún conflicto de competencia o interés.

\author{
Información de los autores \\ Carlos Alarcón Andrade, Médico Tratante de Ginecología Oncológica, Instituto Oncológico Nacional Dr. Juan Tanca \\ Marengo SOLCA, Guayaquil. Ecuador. \\ Nery Rivadeneira Santana, Jefe de Servicio de Ginecología Oncológica, Instituto Oncológico Nacional Dr. Juan \\ Tanca Marengo SOLCA, Guayaquil. Ecuador. \\ Héctor Montez Lainez, Médico Residente de Posgrado, Cirugía General, Instituto Oncológico Nacional Dr. Juan \\ Tanca Marengo SOLCA, Guayaquil. Ecuador. Medico Posgradista Cirugía General, Universidad de Especialidades \\ Espíritu Santo, Samborondón. Ecuador. ORCID: https://orcid.org/0000-0003-3243-456X.
}

\section{Referencias}

1. Vivas $\mathrm{Cl}$, Pantoja M, Holgado A. Adenocarcinoma primario de vulva tipo glándula mamaria. Forma histológica muy infrecuente de neoplasia vulvar. Clínica e Investigación en Ginecología y Obstetricia. 2018;45(4):187-192. doi: 10.1016/i.qine.2017.03.004

2. Di Bonito L, Patriarca S, Falconieri G. Aggressive "breast-like" adenocarcinoma of vulva. Pathol Res Pract. 1992;188(1-2):211-4; discussion 214-6. doi: 10.1016/S0344-0338(11)81181-7. PMID: 1594493.

3. Rogers LJ, Cuello MA. Cancer of the vulva. Int J Gynaecol Obstet. 2018;143 Suppl 2:4-13. doi: $\underline{10.1002 / i j q 0.12609}$

4. Michalski BM, Pfeifer JD, Mutch D, Council ML. Cancer of the Vulva: A Review. Dermatol Surg. 2021;47(2):174-183. doi: 10.1097/DSS.0000000000002584. PMID: $\underline{32947298 .}$

5. Kostov S, Dzhenkov D, Metodiev D, Kornovski Y, Slavchev S, Ivanova Y, et al. A case of human papillomavirus infection and vulvar cancer in a young patient - "hit and run" theory. Gynecol Oncol Rep. 2021;36(100760):100760. doi: 10.1016/i.gore.2021.100760 
6. Serrano B, Brotons M, Bosch FX, Bruni L. Epidemiology and burden of HPV-related disease. Best Pract Res Clin Obstet Gynaecol. 2018 Feb;47:14-26. doi: 10.1016/j.bpobgyn.2017.08.006. Epub 2017 Sep 2 PMID: $\underline{29037457}$.

7. Encalada Salcedo E, Paladines Rodríguez E, Chang Borrero A. Cáncer de vulva: reporte de un caso. Medicina (Guayaquil). 2002;64-6. doi: bvsalud.

8. Desouki MM, Fadare O. Primary adenocarcinomas of the vulva and related structures: An enigmatic and diverse group of tumorsis. Semin Diagn Pathol. 2021;38(1):71-84. doi: 10.1053/i.semdp.2020.09.011. Epub 2020 Sep 25. PMID: 33032903.

9. Kajal B, Talati H, Daya D, Alowami S. Adenocarcinoma apocrino de vulva. Tumores raros. 9 de septiembre de 2013; 5 (3): e40. doi: 10.4081/rt.2013.e40. PMID: 24179652; PMCID: PMC3804815.

10. Rajaram S, Gupta B. Management of Vulvar Cancer. Rev Recent Clin Trials. 2015;10(4):282-8. doi: 10.2174/1574887110666150923112723. PMID: 26411953.

11. Niakan S, Love H, Cao Q, Kawar N. Primary invasive lobular carcinoma arising in mammary-like glands of the vulva managed with neoadjuvant trastuzumab-based chemotherapy, excision, and sentinel lymph node biopsy. Clin Case Rep. 2021;9(1):118-22. doi: $\underline{10.1002 / c c r 3.3475}$

12. Zongo N, Korsaga Somé N, Ouédraogo S, Ouédraogo AS, Zamané H, Zida M, Ouangre E, Sanou A, Niampa $\mathrm{P}$, Lompo OM, Traoré A, Dem A. Cancer de la vulve : stades diagnostiques, traitement et survie dans un pays aux ressources limitées (Burkina Faso) [Cancer of the vulva: Diagnostic stages, treatment and survival in a country with limited resources (Burkina Faso)]. Bull Cancer. 2019 Nov;106(11):1057-1063. French. doi 10.1016/j.bulcan.2019.07.008. Epub 2019 Sep 18. PMID: 31542167.

13. Butler B, Leath CA 3rd, Barnett JC. Primary invasive breast carcinoma arising in mammary-like glands of the vulva managed with excision and sentinel lymph node biopsy. Gynecol Oncol Case Rep. 2013 Sep 12;7:7-9. doi: 10.1016/j.gynor.2013.09.001. PMID: 24624320; PMCID: PMC3895283.

14. Lopes G, DeCesare T, Ghurani G, Vincek V, Jorda M, Glück S, Silva O. Primary ectopic breast cancer presenting as a vulvar mass. Clin Breast Cancer. 2006 Aug;7(3):278-9. doi: 10.3816/CBC.2006.n.041. PMID: $\underline{16942646 .}$

15. de Witte CJ, van de Sande AJ, van Beekhuizen HJ, Koeneman MM, Kruse AJ, Gerestein CG. Imiquimod in cervical, vaginal and vulvar intraepithelial neoplasia: a review. Gynecol Oncol. 2015 Nov;139(2):377-84. doi 10.1016/i.yqyno.2015.08.018. Epub 2015 Aug 31. PMID: 26335596.

16. Levenback CF, Ali S, Coleman RL, Gold MA, Fowler JM, Judson PL, et al. Lymphatic mapping and sentinel lymph node biopsy in women with squamous cell carcinoma of the vulva: a gynecologic oncology group study. J Clin Oncol. 2012 Nov 1;30(31):3786-91. doi: 10.1200/JC0.2011.41.2528. Epub 2012 Jul 2. PMID: 22753905; PMCID: PMC3478573.

17. Al-Mansouri, , Poursoltan P, Simons, Muljono, Boyages. Primary breast cancer of the vulva: A case report and literature review. Journal of Obstetrics and Gynaecology Research. 2018;44(12):1-6. doi: $\underline{10.1111 / \text { iog. } 13778}$

DOI: Digital Object Identifier PMID: PubMeD Identifier SU: Short URL 